\title{
Ultrasound Investigation in Congenital Masseter Hypertrophy
}

\author{
Anida M Băbțan ${ }^{1}$, Bianca A Boșca*2, Maria Crișan ${ }^{2}$ Manuela Lenghel $^{3}$, Radu S Câmpian ${ }^{1}$ and Aranka Ilea $^{1}$ \\ ${ }^{1}$ Department of Oral Rehabilitation, Oral Health and Dental Office Management, Faculty of Dentistry, "Iuliu Haţieganu" University of Medicine and Pharmacy, \\ Cluj-Napoca, Romania
}

${ }^{2}$ Histology Department, Faculty of Medicine, University of Medicine and Pharmacy "Iuliu Haţieganu" Cluj-Napoca, Romania

${ }^{3}$ Radiology Department, Faculty of Medicine, University of Medicine and Pharmacy "Iuliu Haţieganu" Cluj-Napoca, Romania

Received: 制 June 19, 2018; Published: 制 July 05, 2018

*Corresponding author: Bianca Adina Boșca, Department of Histology, Faculty of Medicine, "Iuliu Haţieganu” University of Medicine and Pharmacy, Cluj-Napoca, Romania, Str. L. Pasteur, No. 4, Cluj-Napoca, Romania

\begin{abstract}
Congenital masseter muscle hypertrophy (CMMH) is a developmental disorder characterized by unilateral muscle's overgrowth, resulting in facial asymmetry and dento-maxillary disorders. Although the disease is usually diagnosed by clinical examination, paraclinical investigations such as Computer Tomography (CT), Magnetic Resonance (MR) or ultrasounds (US) are advanced diagnostic methods. This article is the case report of a CMMH in a young male adult, presenting the clinical aspects and using a new diagnostic approach based on US investigation of the masseter muscle.

Keywords: Masseter Muscle; Hypertrophy; Ultrasonography; Diagnosis; Follow-Up
\end{abstract}

Abbreviations: CMMH: Congenital Masseter Muscle Hypertrophy; MR: Magnetic Resonance; US:Ultrasounds; CT: Computer Tomography; TMD: Temporo-Mandibular Disorder; OPT: Ortopantomography

\section{Introduction}

\section{Masseter Muscle Anatomy and Physiology}

The masseter is a thick, quadrilateral muscle, consisting of two parts: superficial and deep. The fibers of the two parts are continuous at their insertion. The superficial part is larger and goes from the zygomatic process of the maxilla and from the anterior two-thirds of the lower border of the zygomatic arch to be inserted into the angle and lower half of the lateral surface of the ramus of the mandible. The deep part is smaller and more muscular in texture. It arises from the posterior $3^{\text {rd }}$ of the lower border and from the entire medial surface of the zygomatic arch. Its fibers pass downward and forward, to be inserted into the upper half of the ramus and the lateral surface of the coronoid process of the mandible. The deep part of the muscle is covered anteriorly by the superficial part; posteriorly, it is covered by the parotid gland [1].

The masseter muscle is involved in mastication, elevation and protrusion of the mandible, functions which develop it into a powerful muscle. The hyperfunction leads to the hypertrophy of the muscle. CMMH is a benign increase in size of the masseter muscle and can be unilateral or bilateral [2] secondary to muscle hypertrophy. The disease involves aesthetic damages, joint disorders, occlusal malfunction, laterodeviation of the mandible. CMMH induces facial asymmetry and, due to the muscle traction towards the affected side, the mandible can be thickened as well [3]. The CMMH etiology is uncertain, although some hypotheses suggest the implication of emotional stress, bruxism or teeth clenching[4].

\section{Diagnostic Approaches}

CMMH diagnosis is usually established based on clinical examinations - inspection and bilateral bimanual masseter palpation. Several studies [5-7] investigated this pathology using a combination of radiological techniques (Magnetic Resonance - MR, Computer Tomography - CT, ultrasound - US) and even histological examination of muscle biopsies. These applications have the advantage of increasing the diagnosis precision in masseter hypertrophy and to correctly differentiate it from other disorders, such as inflammatory disease of the parotid gland (parotiditis), benign or malignant tumors of the muscle or parotid gland, vascular tumors, lipomas or ossifying myositis [8]. Importantly, CMMH must be differentiated from the hemifacial hyperplasia, which, according to Rowe's criteria, involves the enlargement of the viscerocranium - frontal bone without the eye, mandible's lower border, midline of the face and the ear. The hemifacial increase is associated with the enlargement of all the affected tissues, including the teeth [9]. 


\section{Ultrasound in Muscle and Temporomandibular Joint (TMJ) Investigation}

Over the lastyears, a new investigation and monitoring technique has been introduced: ultrasonography. The high-frequency sound waves applications in thorax and abdomen pathologies have been transferred to the cephalic extremity, for a detailed examination the soft tissues, in order to identify and differentiate the malignant tumors from benign lesions (cysts). In a systematic review based on 23 articles, Reis Durãoet al. analyzed the US reliability in measuring the thickness of masseter muscle during relaxation and contraction [10]. Their results showed that the thickness, cross-section, volume and length can be precisely assessed using ultrasonography. In a non-systematic research, Costa et al. investigated the advantage of using strain and shear wave elastography in patients suffering from myofascial temporomandibular disorder (TMD) pain [11]. Their findings indicated that shear elastography showed increased hardness in affected masseter muscles compared with healthy ones. When using shear wave elastography, the results showed increased elasticity in TMD affected muscles.

A study [12] which investigated masseter muscle thickness in unilateral partial edentulous patients during resting and contraction using an US transducer with high frequency (7.5-10 $\mathrm{MHz}$ ) reported muscle atrophy on the edentulous side. GonzálezArriagada et al. used Doppler US to analyze intramasseteric nodules with similar structure, in order to find more specific diagnostic method and to differentiate the nodules from other lesions [13].
The above-mentioned studies suggest that US could be considered as a useful additional non-invasive approach for investigating and monitoring both solid and liquid-full structures. The purpose of the present paper is to report a case of unilateral CMMH and to assess the efficacy of a non-invasive investigation based on ultrasound for both the diagnosis and the patent's follow-up.

\section{Materials and Methods}

\section{Case Report}

A 31years-old male was referred to the Oral Rehabilitation, Oral Health and Dental Office Management Department in April 2013 for discomfort in the posterior right mandibular angle. He was examined by clinical, photographic and radiographic (Ortopantomography - OPT) investigations. His medical history included: mumps at 4 years old, a tonsillectomy at 5 years old, nose injury followed by nasal septum deviation at 7 years old, and infection with hepatitis $\mathrm{C}$ virus (HCV), diagnosed in 2003. The patient was a tobacco smoker and was under no medical treatment. The clinical examination revealed a facial asymmetry due to the hypertrophy of the masseter muscle on the right side, hyperpilosity on the right jaw region, with the centrifugal hair growth, and a $5 \mathrm{~mm}$ laterodeviation to the right side of the nasal pyramid. For a better historical report of the facial asymmetry, pictures from the patient's childhood and adolescence were included (Figure 1). Written consent for publishing pictures without preserving patient's identity was obtained.

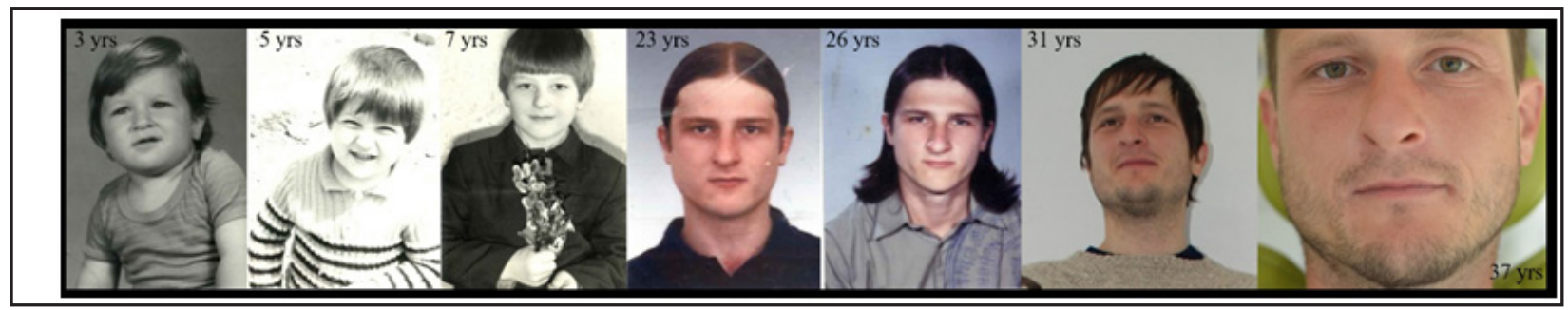

Figure 1: Facial asymmetry preserved with aging.

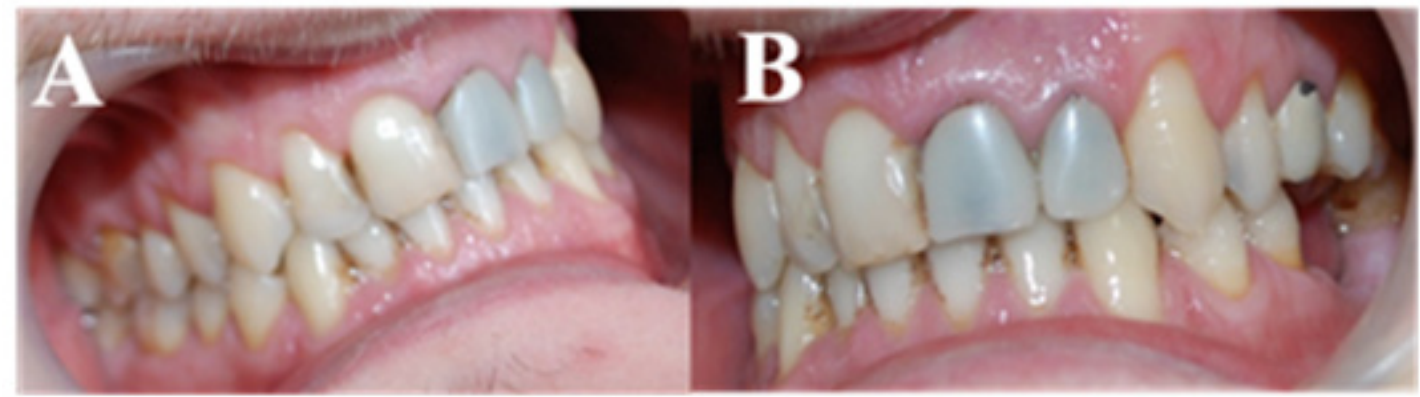

Figure 2: Right (A) and left (B) side oral aspect: mandibular partial edentulous (Kennedy class III edentation); midline deviation to the right.

The right basilar and angular regions of the mandible were thickened and embattled compared with the left side. The palpation produced low intensity pain on the right retroangular region, submandibular region at the lower border. Additionally, the right condylar region and the posterior vertical mandibular ramus were painful. During mandibular movements, especially mouth opening, theright TMJ (Temporomandibular joint) clicking and pain occurred, at the beginning of the mouth opening. Oral examination (Figure 2) revealed a $3 \mathrm{~mm}$ right laterodeviation of the mandible, premature contacts between 13 and 44 frontal teeth, a bayonet 
guiding of the mandible in propulsion, and laterality movement guided by 12, 31, 41, 42 teeth.OPT measurements (Figure 3) showed mandibular asymmetrical rami. Measurements of the facial bones were reported to a horizontal line designed through ANS (Anterior Nasal Spine) point. The horizontal mandibular body was measured from the midline to the distal extremity, the maxillary was measured from the midline, above the roots of the superior teeth, to the distal extremity of the tuberosity; the antero-posterior distance between the condyle and coronoid apophyses was measured from the coronoid's peak perpendicular to the condyle.

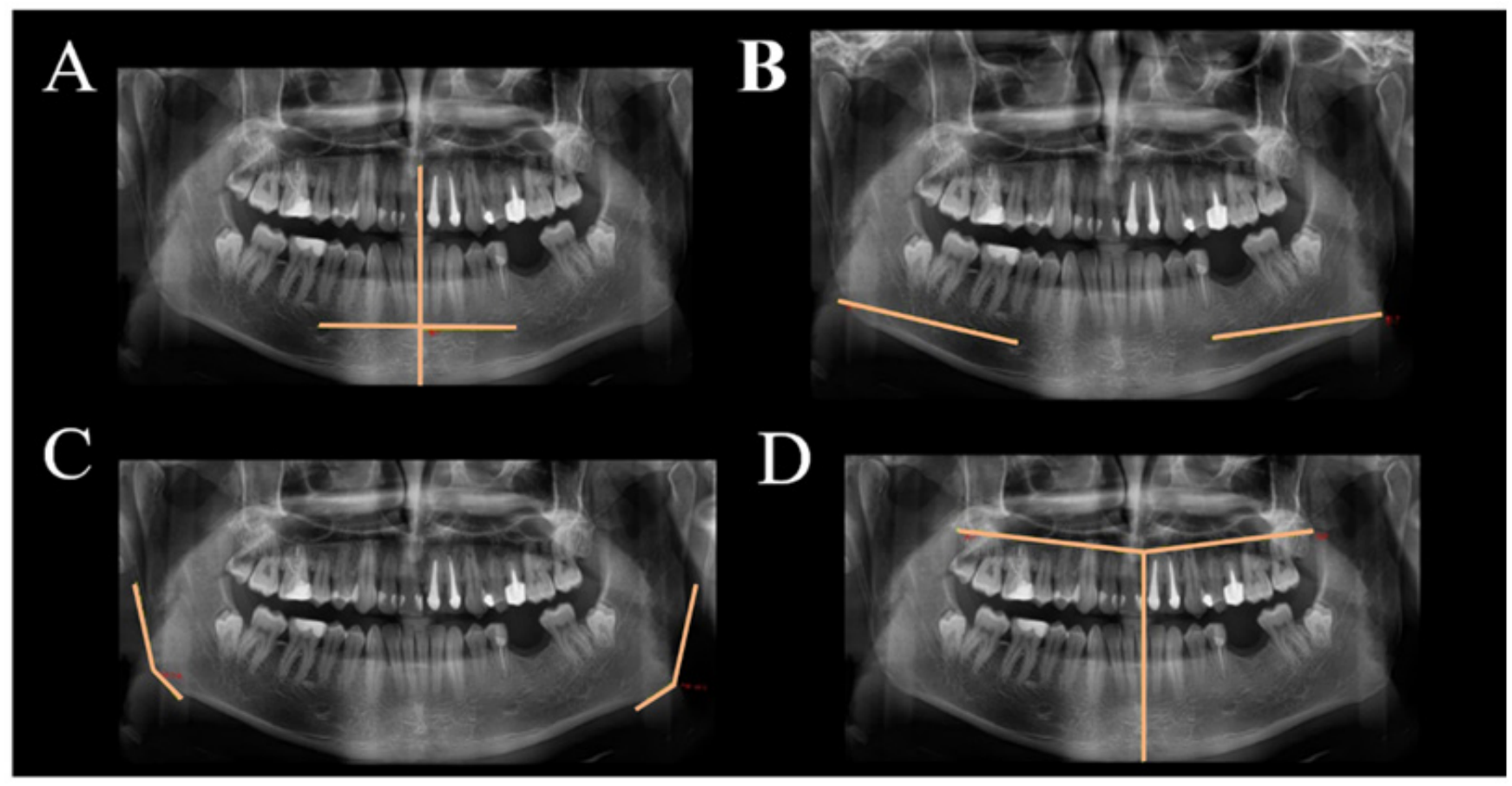

Figure 3: OPT measurements of the mandible and maxilla: (A) mandibular body from midline to the emergence of the mental foramen; (B) mandibular body from the emergence of the mental foramen to the distal extremity of the body; (C) measurement of mandibular angulations delimited between the tangents to the body and the rami; (D) maxilla length measured from the midline to the distal extremity of tuberosities.

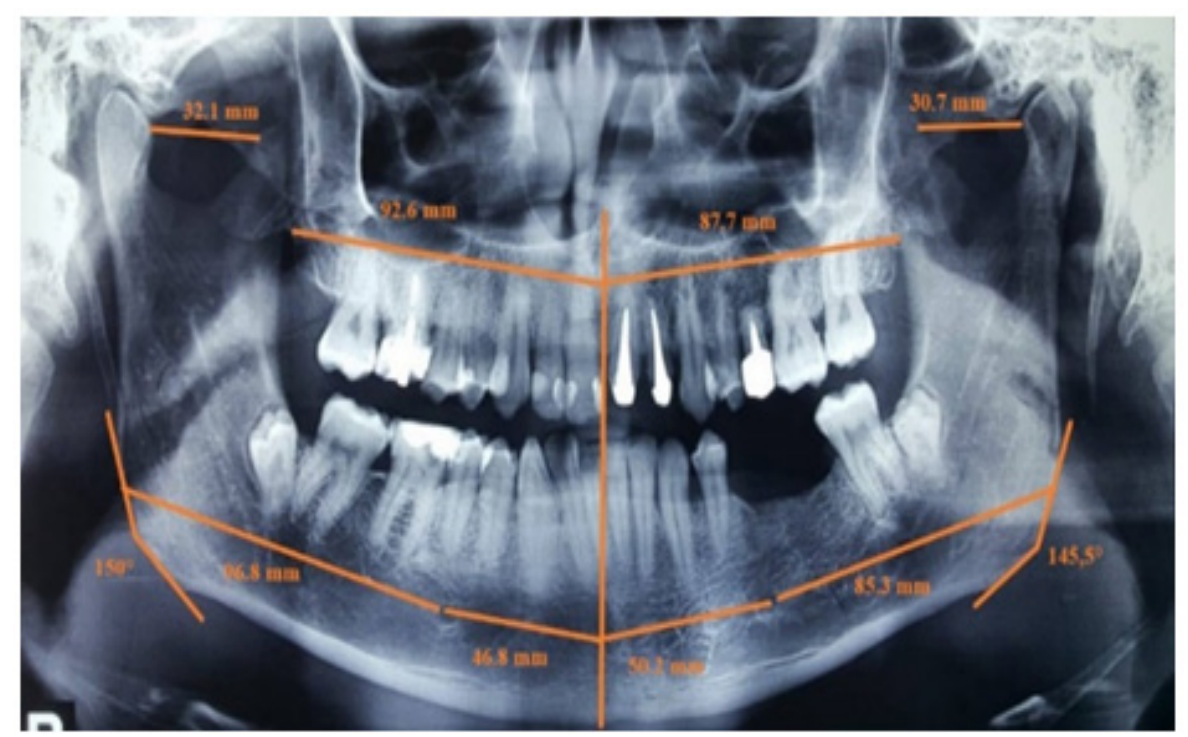

Figure 4: Facial bone measurements on OPT after 5 years.

Tangents to the mandibular body and rami were drawn and the angulations of the junctions were measured. The results showed an increased size of the right part of the mandible and maxilla and the right condyle, compared with the left side. Based on the clinical examinations and the OPT measurements, the diagnosis of right CMMH was established. The patient returned in 2018 to the Oral Rehabilitation, Oral Health and Dental Office Management Department, requesting dental and prosthetic restorations. The 
clinical examinations, photostatic and OPT (Figure 4) investigations were repeated. Limbs length were measured and summarized in Table 1. The measurements on the OPTs taken in 2013 and 2018 were presented in Table 2. Paraclinical investigations were supplemented with an US examination. Measurements were performed with the patient sitting in the dentist chair, with the mandible resting, in centric point and open mouth. A L64 linear array transducer (18-5
MHz) with stand-off device (Arietta, Hitachi, Ltd. 2013, 2017, Q1EEZ1295) was used. The linear transducer was positioned at the level of the occlusal plane and in a parallel orientation, overlying the masseter muscle. Measurements regarding the thickness of the epidermis, dermis, masseter muscle and the anatomical parts of TMJ (Figures 5 \& 6), were registered (Table 3).

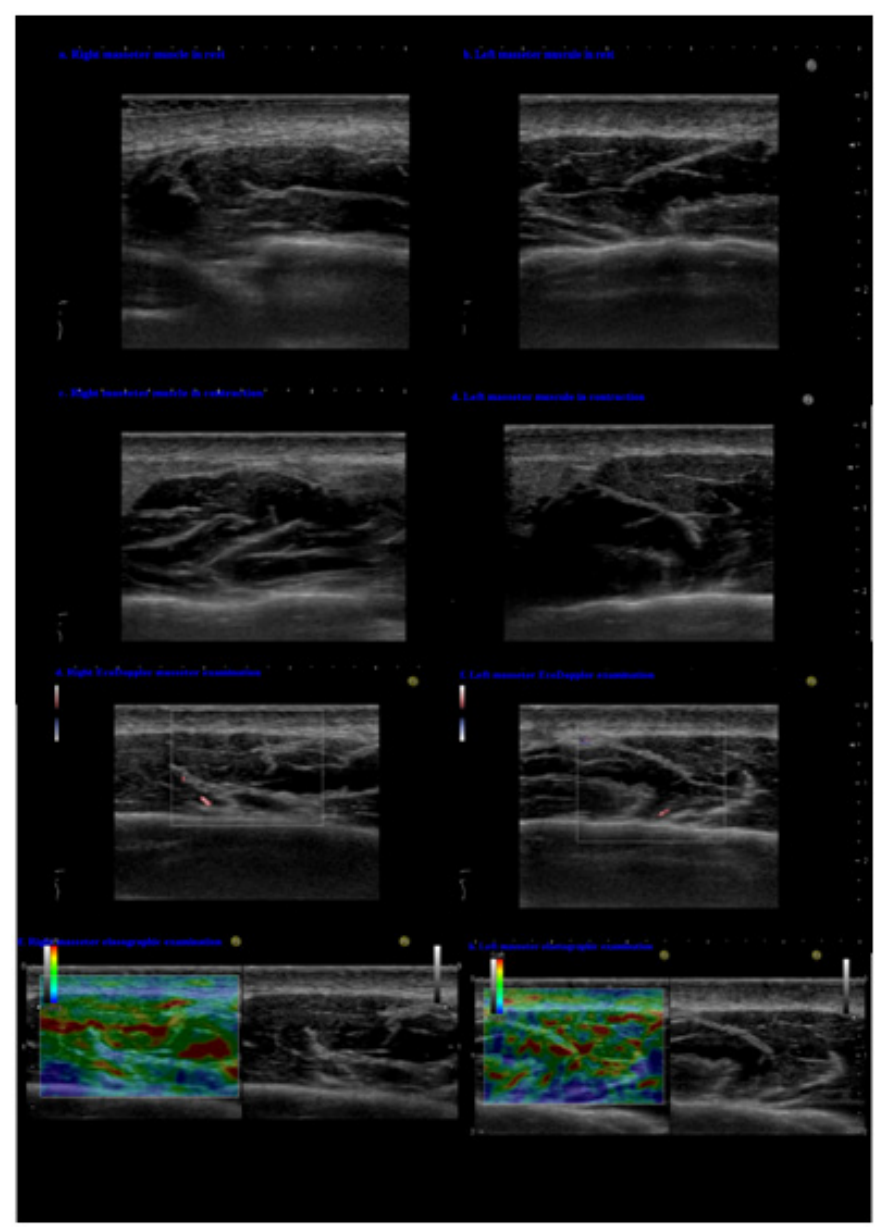

Figure 5: US masseter muscle examination: comparative right and left transverse sections of the masseter muscle - hypoechoic in resting and contraction (a-d) and the anterior portion of parotid gland - moderately echogenic, limited inferiorly by a hyperechoic lamella, which is the inferior border of the mandibular body. Doppler mode (e, f) showed a slightly higher vascularization (red) on the right masseter muscle; Dual US image (elastography-grayscale) of the masseter shows heterogenous mosaic aspect on both sides, but with a predominant average green on the right side, suggesting a stiffer right masseter muscle.

Table 1: Right and left anatomical measurements.

\begin{tabular}{|c|c|c|}
\hline Anatomical parts & Right (mm) & Left (mm) \\
\hline Arm & 78 & 17.5 \\
\hline Leg & 103 & $27.5 / 10.5$ \\
\hline Foot & $27.5 / 10.5$ & $6.5 / 4.4$ \\
\hline Ear & $6.9 / 4.6$ & \\
\hline
\end{tabular}

Comparison between the left and right masseter areas was performed. Masseter's vascular flow was assessed using Doppler ultrasound function (Figure 5e \& 5f). Muscle's hardness was analyzed using US elastographic mode (Figure $5 \mathrm{~g} \& 5 \mathrm{~h}$ ).The clinical re-evaluation after five years indicated no worsening in the patient's condition. Palpation induced pain at the right TMJ. There were differences of $0.4-0.5 \mathrm{~mm}$ between right and left patient's upper limbs and ears. The occlusion showed no modification, although the left lower second premolar tooth was removed in 2015. The analysis of the OPTs taken in 2013 and 2018 comparing the measurements between the right and left side (Table 2) showed that the right maxillary bone, mandible, condyle, and coronoid 
were asymmetrically enlarged.US examinations of the TMJs (Figure 6) confirmed and completed the OPT measurements regarding the asymmetry. US investigations of the masseter muscles showed (Figure 5) that the right side muscle was enlarged with $2.8 \mathrm{~mm}$ compared with the left side, as well as the covering dermis - 0.5 $\mathrm{mm}$. Doppler function mode evidenced higher quantitative arterial vascularization of the right masseter muscle. The elastography of the right masseter muscle showed a homogenous green area with small red areas, suggesting a harder muscle compared with the left masseter, where an inhomogeneous green and red area was seen, suggesting a softer muscle.

Table 2: Bone segments measurements on OPT after five years.

\begin{tabular}{|c|c|c|c|c|c|c|c|c|}
\hline \multirow[b]{3}{*}{ Bone segment } & \multicolumn{8}{|c|}{ OPT measurements (mm) } \\
\hline & \multicolumn{3}{|c|}{2013} & \multicolumn{3}{|c|}{2018} & \multirow{2}{*}{$\begin{array}{l}\text { Increased } \\
\text { right side } \\
\text { after } 5 \text { yrs }\end{array}$} & \multirow{2}{*}{$\begin{array}{c}\text { Increased left } \\
\text { side } \\
\text { after } 5 \text { yrs }\end{array}$} \\
\hline & Right & Left & Difference & Right & Left & Difference & & \\
\hline $\begin{array}{l}\text { Mandibular angle } \\
\text { (degrees) }\end{array}$ & 148 & 136.5 & 11,5 & 150 & 145.5 & 4.5 & 2 & 9 \\
\hline $\begin{array}{l}\text { Mandibular } \\
\text { horizontal branch }\end{array}$ & 104.9 & 102.6 & 2.3 & 116.14 & 109.16 & 6.98 & 11.5 & 6.56 \\
\hline Maxillary lenght & 74.4 & 69.06 & 5.34 & 74.89 & 70.9 & 3.99 & 0.89 & 1.84 \\
\hline $\begin{array}{l}\text { Antero-posterior } \\
\text { distance } \\
\text { between condyle } \\
\text { and coronoid } \\
\text { apophyses }\end{array}$ & 30.3 & 26.4 & 3.9 & 32.1 & 30.7 & 1.4 & 1.8 & 4.3 \\
\hline
\end{tabular}

Table 3: US measurements at different anatomical levels.

\begin{tabular}{|c|c|c|c|}
\hline & Right & US measurements (mm) & Difference between right and left \\
\hline TMJ $^{*}$ & $13 / 3.7$ & $7.9 / 2.3$ & $4.1 / 1.4$ \\
\hline $\begin{array}{c}\text { Masseter muscle thickness in } \\
\text { contraction }\end{array}$ & 17.4 & 15.6 & 1.8 \\
\hline $\begin{array}{c}\text { Masseter muscle thickness in } \\
\text { resting }\end{array}$ & 12.9 & 10.1 & 2.8 \\
\hline Epidermis layer thickness & 1.7 & 2.1 & -0.4 \\
\hline Dermis layer thickness & 2.4 & 1.9 & 0.5 \\
\hline
\end{tabular}

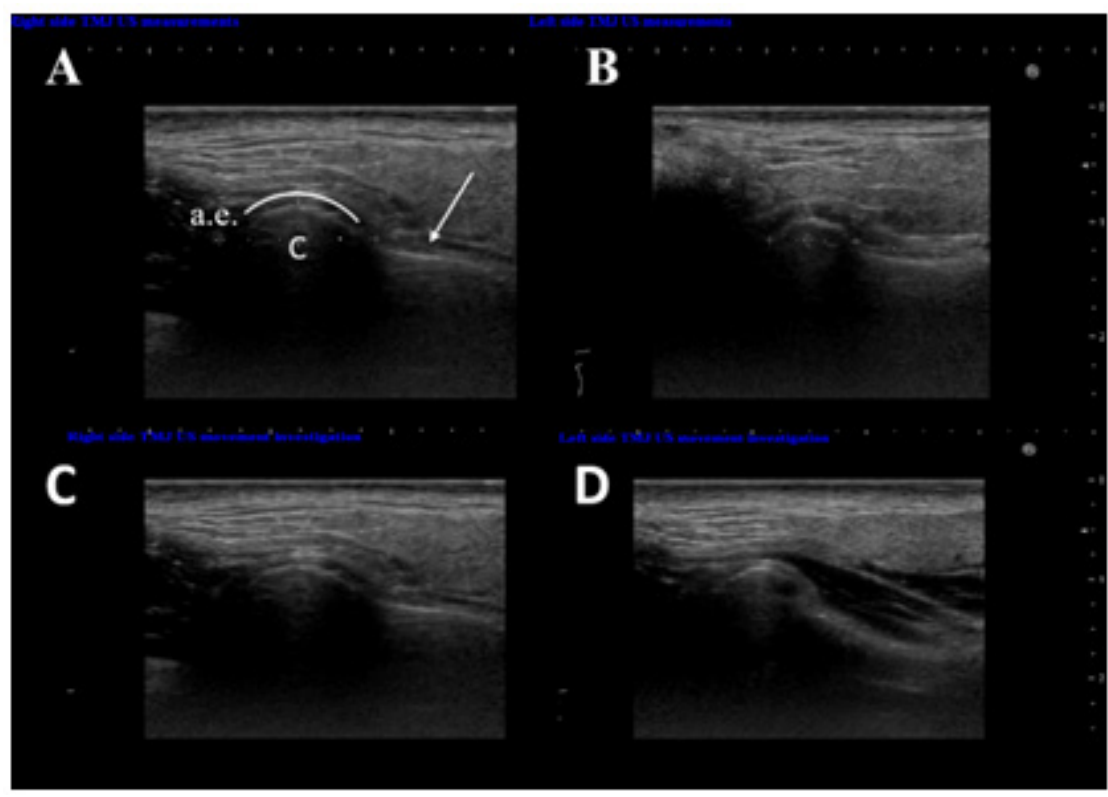

Figure 6: US TMJ examination: comparative right and left transverse sections of the TMJ: the hypoechoic longitudinal area (white arrow) representing the pterygoid muscle, c - condyle, a.e. - articular eminence, and the disc with the joint space - the vaulted white line. The two TMJs had different sizes during resting (in point centric - A and B) and movement (mouth opening at $3 \mathrm{~cm}-\mathrm{C}$ and D). 


\section{Discussion}

CMMH is characterized by unilateral or bilateral enlargement of the masseter muscles, of unknown etiology. CMMH affects both males and females, usually after puberty [3], with the mean at early adulthood, about 30 years of age [14]. Contrarily to these statistics, in the present case report the disease began in early childhood, as seen in Figure 1. Anthropometrical measurements performed at the age of 37 and correlated with the OPT and US suggested a continuous growth of the maxilla, mandible, and TMJs. The OPT revealed the inclusion of both lower third molars. Even though there was a slow continuous increase of the mandible, especially on the right side, there still was no sufficient eruptive space for the third molars. This might be due to the fact that the dimensional changes occurred in all three spatial axes of the mandibular ramus. The comparative radiological measurements showed a $9^{\circ}$ increase of the left mandible angle, which could explain the 4.3 $\mathrm{mm}$ increase in the antero-posterior distance between condyle and coronoid apophyses, the wider angle induced the TMJ changes. During the initial clinical examination, the patient accused pain in the retroangular, submandibular region at the lower border, right condylar region and posterior third vertical mandibular ramus.

After 5 years, the pain was in remission, affecting only the TMJ area. Possibly, the discomfort and pain were caused by the continuous reshape of the hard and soft tissues, since the trigger points corresponded to the muscle's insertions on the bone. In time, the muscular-ligament system adapted and the symptoms decreased. Ultrasonographic measurements revealed a thicker skin on the left side compared with the right hypertrophied egion. The difference could be a mechanism to compensate the reduced dimension of the left masseter muscle. The left side smaller dimensions could have been induced by their inactivity and the consequent hypotrophy, due to right side hyperactivity. Associated with the differences in the facial soft tissue aspect, the summed data could indicate a hemifacial hemihypertrophy. However, this condition has more prominent clinical features regarding the facial asymmetry. The etiology of the hemifacial hemihypertrophy includes chromosomal alterations, abnormalities in cell differentiation, prenatal disorders or endocrine dysfunctions [7].

An innovative approach in CMMH diagnosis would be the genetic assay analyzing the affected muscle cell versus a healthy one. Early stage intervention could prevent the disease evolution or dento-maxillary complications. Recent advances in facial recognition software could be a helpful non-invasive technique for the early diagnosis and classification of facial asymmetry, regarding the hard and soft tissues, and in consequence a minimal invasive treatment strategy. Maillard's reaction final products Advanced glycation end products (AGEs) are glycated proteins with exogenous and endogenous sources, deposited in human tissues in a time and dose dependent manner. AGEs bind to collagen fibers and thus these compounds can be assesed in skin using histological, imunohistochemical or radiological techniques such as ultrasonography [15]. AGEs have negative effects by generating oxidative stress and local and general inflammation; binding to collagen fibers, AGEs lead to collagen dissolution, and compromise the integrity and physiology of anatomical structures [16]. Besides the role in chronic inflammation, AGEs implication in genetic and autoimmune diseases has been demonstrated by several studies.

The AGEs accumulation in neural network has been reported in patients with Huntington's disease (HD) $[17,18]$, who showed significantly higher positivity for AGEs receptors - RAGE, compared with the healthy controls. Maher suggested that methylglyoxal-MG, the main AGEs precursor, alongside with genetic vulnerabilities could also induce mitochondrial dysfunction and the development of autism [19]. Campos et al. assessed the oxidative biomarkers in adolescents and adults with Down syndrome [20]. They found that AGEs, dityrosine (diTyr), hydrogen peroxide $\left(\mathrm{H}_{2} \mathrm{O}_{2}\right)$ and nitrite/ nitrate (NOx) had higher levels in Down syndrome patients versus healthy subjects. These results could support the hypothesis that AGEs are one of the etiological factors in congenital masseter hypertrophy. A clinical study analyzed AGEs accumulation in the affected muscle fibers compared to healthy cells using US; this could confirm this hypothesis. In this case report, US proved to be a useful complementary investigation. It confirmed the findings of the clinical examination and the OPT measurements.

Moreover, the Doppler and elastography modes have the advantage to reveal the differences regarding the masseter muscle vascular flow and stiffness. The results were influenced by factors such as anthropometric parameters, the muscle anatomy and position, the transducer characteristics and positioning, and the associated diseases [11]. CMMH treatment [21] usually consists of non-invasive approaches: tranquilizers, intramuscular administration of botulinum toxin type A - muscle detensioner - with the mention that in time the substance becomes inactive and the injection should be repeated after 4-6 months; dental treatments and re-treatments, removal of occlusal interferences, mouthguards for the balanced occlusion. The surgical treatment includes selective mandible basilar and angle ostectomies, soft tissue excision, orthognathic surgery, and reconstructive procedures [22].

\section{Conclusion}

CMMH is a benign pathology associated with significant facial asymmetry and TMJ disorders. As a non-invasive, painless, no radiation emission paraclinical investigation, US could be a useful approach for CMMH diagnosis and evolution, as well as for the posttherapeutic follow-up or the monitoring of complications. Further studies are needed to elaborate a US examination protocol in head and neck muscle pathology, and also in TMJ pathologies.

\section{Acknowledgement}

This study was supported by "Iuliu Haţieganu" University of Medicine and Pharmacy Cluj-Napoca, PhD Grant no 3999/01.10.2016, and partially by the COFUND-ERA-HDHL ERANET Project, European and International Cooperation Subprogram 3.2 - Horizon 2020, PNCDI III Program - Biomarkers for Nutrition and Health - "Innovative technological approaches for validation of salivary AGEs as novel biomarkers in evaluation of risk factors in diet-related diseases", grant no 25/1.09.2017. 


\section{References}

1. Papilian V (1982) Anatomiaomului. ( $6^{\text {th }}$ Edition) Vol. I. Edited by Editura didactică și pedagogică București, București.

2. Waldhart E, Lynch JB, John B (1971) Benign hypertrophy of the masseter muscles and mandibular angles. Arch Surg 102(2): 115-118.

3. Sannomya EK, Gonçalves M, Cavalcanti MP (2006) Masseter muscle hypertrophy: case report. Braz Dent J 17(4): 347-350.

4. Black MJ, Scholss MD (1985) Masseteric muscle hypertrophy. J Otolaryngol 14(3): 203-205.

5. Morse MH, Brown EF (1990) Ultrasonic diagnosis of masseteric hypertrophy. Dentomaxillofac Radiol 19(1): 18-20.

6. Newton JP, Cowpe JG, McClure IJ, Delday MI, Maltin CA (1999) Masseteric hypertrophy: Preliminary report. Br J Oral Maxillofac Surg 37(5): 405408.

7. Miranda RT, Barros LM, Santos LA, Bonan PR, Martelli H (2010) Clinical and imaging features in a patient with hemifacial hyperplasia. J Oral Sci 52(3): 509-512.

8. Da Silva K, Mandel L (2006) Bilateral temporalis muscle hypertrophy: a case report. Oral Surg Oral Med Oral Pathol Oral Radiol Endod 102(1): e1-3.

9. Rowe NH (1962) Hemifacial hypertrophy. Review of the literature and addition of four cases. Oral Surg Oral Med Oral Pathol 15: 572-587.

10. Reis Durão AP, Morosolli A, Brown J, Jacobs R (2017) Masseter muscle measurement performed by ultrasound: a systematic review. DentomaxillofacRadiol 46(6): 20170052.

11. Costa YM, Ariji Y, Ferreira DMAO, Bonjardim LR, Conti PCR, Ariji E, et al (2018) Muscle hardness and masticatory myofascial pain: assessment and clinical relevance. J Oral Rehabil.

12. Sathasivasubramanian S, Venkatasai PM, Divyambika CV, Mandava R, Jeffrey R, et al. (2017) Masseter Muscle Thickness in Unilateral Partial Edentulism: An Ultrasonographic Study. J Clin Imaging Sci 7: 44.

\section{ISSN: 2574-1241}

DOI: 10.26717/BJSTR.2018.06.001349

Bianca Adina Boșca. Biomed J Sci \& Tech Res

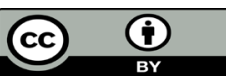

This work is licensed under Creative Commons Attribution 4.0 License

Submission Link: https://biomedres.us/submit-manuscript.php
13. González-Arriagada WA, Santos-Silva AR, Vargas PA, Lopes MA (2017) Diagnostic approach to intramasseteric nodules. Oral Surg Oral Med Oral Pathol Oral Radiol 123(1): e16-e21.

14. Riefkohl R, Georgiade GS, Georgiade NG (1984) Masseter muscle hypertrophy. Ann Plast Surg 12(6): 528-532.

15. Crisan M, Taulescu M, Crisan D, Cosgarea R, Parvu A, et al. (2013) Expression of Advanced Glycation End-Products on Sun-Exposed and Non-Exposed Cutaneous Sites during the Ageing Process in Humans. PloS One 8(10): e75003.

16. IleaA, Băbțan AM, Boșca BA, Crișan M, Petrescu NB, et al. (2018) Advanced glycation end products (AGEs) in oral pathology. Arch Oral Biol 93: 22-30.

17. Ma L, Nicholson LF (2004) Expression of the receptor for advanced glycation end products in Huntington's disease caudate nucleus. Brain Res 1018(1): 10-17.

18. Anzilotti S, Giampà C, Laurenti D, Perrone L, Bernardi G, Melone MA, et al. (2012) Immunohistochemical localization of receptor for advanced glycation end (RAGE) products in the R6/2 mouse model of Huntington's disease. Brain Res Bull 87(2-3): 350-358.

19. Maher P (2012) Methylglyoxal, advanced glycation end products and autism: Is there a connection? Med Hypotheses 78(4): 548-552.

20. Campos C, Guzmán R, López-Fernández E, Casado A (2011) Evaluation of urinary biomarkers of oxidative/nitrosative stress in adolescents and adults with Down syndrome. BiochimBiophys Acta 1812(7): 760-768.

21. Ayhan M, İşler SC, Kasapoglu C (2018) Combination of Medical and Surgical Treatments for Masseter Hypertrophy. Case Rep Dent 2018: 7168472.

22. Nandimath S, Rajkumar GC, Nayak T, Ashwin DP, Rudresh KB (2016) Hemifacial hypertrophy: Exploring new avenues of treatment modalities. Natl J of Maxillofac Surg 7(1): 100-104.

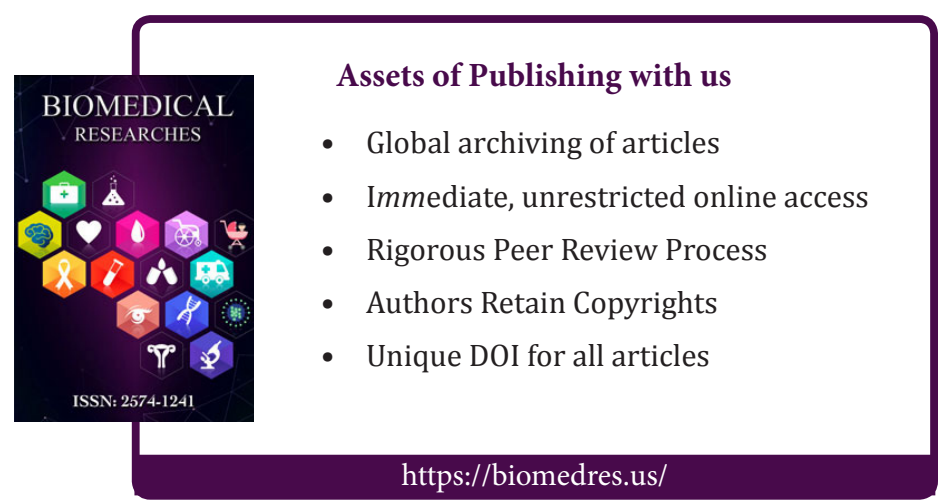

